\title{
Hypoxia causes connexin 43 internalization in neonatal rat ventricular myocytes
}

\author{
Asaf Danon ${ }^{1,2}$, Naama Zeevi-Levin ${ }^{3}$, Dani Y. Pinkovich ${ }^{4}$, Tomer Michaeli ${ }^{4}$, Alexander \\ Berkovich ${ }^{4}$, Moshe Flugelman 5 , Yonina C. Eldar ${ }^{4}$, Michael R. Rosen ${ }^{6}$ and Ofer Binah ${ }^{1,2,3}$ \\ ${ }^{1}$ Department of Physiology, Ruth and Bruce Rappaport Faculty of Medicine, Technion-Israel Institute of Technology, Haifa, Israel \\ ${ }^{2}$ Rappaport Family Institute for Research in the Medical Sciences, Haifa, Israel \\ ${ }^{3}$ The Sohnis Family Stem Cells Center, Ruth and Bruce Rappaport Faculty of Medicine, Haifa, Israel \\ 4 The Faculty of Electrical Engineering, Technion-Israel Institute of Technology, Haifa, Israel \\ ${ }^{5}$ Department of Cardiology, Carmel Medical Center, Haifa, Israel \\ ${ }^{6}$ Departments of Pharmacology and Pediatrics and Center for Molecular Therapeutics, College of Physicians and Surgeons \\ of Columbia University, New York, USA
}

\begin{abstract}
Gap junctions produce low resistance pathways between cardiomyocytes and are major determinants of electrical conduction in the heart. Altered distribution and function of connexin 43 (Cx43), the major gap junctional protein in the ventricles, can slow conduction, and thus contribute to arrhythmogenesis in experimental models such as ischemic rat heart and pacing-induced atrial fibrillation. The mechanisms underlying reduced gap junctional density and conductance during ischemia may involve decreased Cx43 synthesis, increased degradation and/or Cx43 migration into areas which do not contribute to intercellular communication. To test more rigorously the hypothesis that hypoxia resulting from ischemia causes $\mathrm{Cx} 43$ internalization, we infected neonatal rat ventricular myocytes (NRVM) with a Cx43-GFP chimera, which enabled us to investigate by means of confocal microscopy the effect of hypoxia $\left(1 \% \mathrm{O}_{2}\right.$ for $5 \mathrm{~h}$ ) on $\mathrm{Cx} 43$ distribution in live cardiomyocytes. Importantly, this protocol permitted each culture to serve as its own control. To this end we used life confocal microscopy analysis to determine in the same pair of myocytes the effects of hypoxia on Cx43-GFP distribution at the gap junctional (GJ) and non-GJ areas. In support of this hypothesis, we found that compared to normoxia, $5 \mathrm{~h}$ of hypoxia reduced the Cx43-GFP signal at the GJ areas (defined as the border area) and caused a corresponding increase in the Cx43-GFP signal at the non-border areas, thus providing an additional explanation for the intercellular uncoupling during ischemic conditions.
\end{abstract}

Key words: Gap junctions - Connexin 43 - Cardiac myocytes - Hypoxia

\begin{abstract}
Abbreviations: Cx43, connexin 43; NVRM, neonatal rat ventricular myocytes; GFP, green fluorescence protein; GJ, gap junctional.
\end{abstract}

\section{Introduction}

Gap junctions interconnecting adjacent cardiomyocytes form low resistance electrical communications, which enable propagation of the action potential throughout the complex cardiac

Correspondence to: Ofer Binah, Department of Physiology, Ruth and Bruce Rappaport Faculty of Medicine, Technion-Israel Institute of Technology, P.O.Box 9649, Haifa 31096, Israel

E-mail: binah@tx.technion.ac.il structure. Thus, along with excitable features, gap junctions constitute important determinants of the coordinated mechanical performance of the myocardium. Due to the central role of connexin $43(\mathrm{Cx} 43$, the major gap junctional protein in the ventricles) in conduction, alterations in their distribution and function can cause arrhythmogenesis, as demonstrated in several experimental models such as hypertrophy, ischemic rat heart (Rohr et al. 1998; Beardslee et al. 2000; Saffitz et al. 2000), and pacing-induced atrial fibrillation (Polontchouk et al. 2001; Ausma et al. 2003; Sosunov et al. 2005; Chaldoupi 
et al. 2009). In these settings, decreased gap junctional conductance can slow conduction velocity thereby contributing to initiation and/or perpetuation of arrhythmias (Spach et al. 2000; Rohr 2004; Zeevi-Levin et al. 2005). The mechanisms responsible for decreased gap junctional conductance during ischemia may involve decreased $\mathrm{Cx} 43$ synthesis, increased degradation and/or Cx43 migration into areas which do not contribute to intercellular communication.

Several studies have investigated the mechanisms responsible for decreased conduction velocity during ischemia/ hypoxia (Beardslee et al. 2000; Turner et al. 2004). Ex vivo ischemia was induced by stopping perfusion of the Langendorff-perfused rat heart, and in vitro, cardiomyocytes cultures were exposed to hypoxia and diminished nutrients supply (e.g., Turner et al. 2004; Zeevi-Levin et al. 2005). In this regard, we exposed neonatal rat ventricular myocytes (NRVM) to 5 h of hypoxia $\left(1 \% \mathrm{O}_{2}\right)$, and found that conduction velocity was decreased by $\sim 20 \%$. Concomitantly, Cx 43 protein content in whole cell lysates was decreased by $\sim 50 \%$ and the $\mathrm{Cx} 43$ immunofluorescence signal decreased similarly (Zeevi-Levin et al. 2005). Other studies that showed comparable gap junctional electrical uncoupling by hypoxia but no decrease in $\mathrm{Cx} 43$ protein level, suggested that the underlying mechanism is a shift from the phosphorylated to the nonphosphorylated form of $\mathrm{Cx} 43$ as well as internalization of the channel protein (Beardslee et al. 2000; Turner et al. 2004). In these studies total $\mathrm{Cx} 43$ protein content was not changed by hypoxia, but the immunofluorescence $\mathrm{Cx} 43$ signal at the gap junctions was decreased. A weakness of these experiments was that separate observations were made on control and hypoxic cultures, and the results were inconclusive.

To test more rigorously the hypothesis that hypoxia causes Cx43 internalization, we infected NRVM with a GFP-tagged Cx43 (Cx43-GFP) chimera, which enabled us to investigate by means of confocal microscopy the effect of hypoxia on Cx43 distribution in living cardiomyocytes. Importantly, this protocol permitted each culture to serve as its own control. As will be demonstrated, hypoxia caused migration of $\mathrm{Cx} 43$ away from the gap junctional areas into the cell interior, which may contribute to attenuated intercellular coupling during ischemia.

\section{Materials and Methods}

The research conforms to the Guide for the Care and Use of Laboratory Animals published by the US National Institutes of Health (NIH publication No. 85-23; revised 1996).

\section{Cultures of neonatal rat ventricular myocytes (NRVM)}

NRVM cultures were prepared as previously described (Rubin et al. 1995), with minor modifications. In brief, ventricles from 1-2 day-old Sprague-Dawley rats were dissociated enzymatically at room temperature, using the protease RDB (catalog no. 300-0; IIBR, Israel). Myocytes were collected following 10-12 cycles of 10 min digestion, and pooled cells were re-suspended in growth medium: Ham's F10 supplemented with $5 \%$ fetal calf serum, 5\% horse serum, $100 \mathrm{U} / \mathrm{ml}$ penicillin, $100 \mathrm{mg} / \mathrm{ml}$ streptomycin (Biological Industries, Bet-Haemek, Israel) and $1 \mathrm{mM} \mathrm{CaCl}_{2}$ (up to a final concentration of $1.3 \mathrm{mM}$ ). To reduce fibroblast content, cell suspensions were pre-plated on culture flasks for $1 \mathrm{~h}$ in the incubator, and $5 \mathrm{mg} / 100 \mathrm{ml}$ bromodeoxyuridine (BrdU; Sigma) were added to the medium. Subsequently, myocytes were seeded on micro-electrode-array (MEA) plates or on 6-cm Petri dishes pre-coated with collagen type I (Sigma, C-8919) diluted $1: 10$ in acetic acid, at a density of $10^{4}$ and $10^{6}$ cell $/ \mathrm{mm}^{2}$, respectively. The cultures were maintained in a humidified incubator, with $5 \% \mathrm{CO}_{2}+95 \%$ air, at $37^{\circ} \mathrm{C}$. Unsettled cells were washed out after $24 \mathrm{~h}$ and the medium replaced, and then replaced again on alternating days. Cultures were maintained in the incubator 5 days prior to data recordings.

\section{Extracellular electrograms recordings using the MEA data acquisition system}

Unipolar electrograms were recorded from NRVM seeded on MEA plates (Multi Channel Systems, Reutlingen, Germany) as previously described (Meiry et al. 2001; Zeevi-Levin et al. 2005; Reisner et al. 2009). In brief, the MEA plate contains an $8 \times 8$ matrix of 60 titanium-nitride electrodes $(30 \mu \mathrm{m}$ diameter) embedded in its center, with an inter-electrode distance of $200 \mu \mathrm{m}$. For the electrophysiological measurements, MEA plates were removed from the incubator, placed in the recording apparatus preheated to $37^{\circ} \mathrm{C}$. Spontaneous electrical activity of the NRVM culture was recorded within 5 min after removal from the incubator. Electrogram analysis was performed automatically using custom-made MATLAB $\left(\right.$ MATLAB $^{\mathrm{R}}$ 6.5) routines (Meiry et al. 2001; Zeevi-Levin et al. 2005; Reisner et al. 2009). The local activation times (LAT) at each electrode, defined as the time of occurrence of the first derivative plot minimum of the fast activation phase, was used to construct activation maps and calculate conduction velocity. The scalar value of local conduction velocity was calculated at each of the array electrodes as previously described (Meiry et al 2001; Zeevi-Levin et al. 2005; Reisner et al. 2009). The value of the conduction velocity presented for each measurement was taken as the mean value of local velocities of all 60 electrodes.

\section{Exposing NRVM cultures to hypoxia}

Five-day-old NRVM cultures seeded on 6-cm Petri dishes were photographed using laser confocal microscopy (see 
details below) before, and after introduction into the hypoxia chamber (Billups-Rothenberg Inc., Del Mar, CA, USA). A hypoxic atmosphere was generated by flushing the chamber with a pre-analyzed gas mixture of $94 \% \mathrm{~N}_{2}, 5 \% \mathrm{CO}_{2}$, and $1 \% \mathrm{O}_{2}$. The incubator temperature was $37^{\circ} \mathrm{C}$. The exposure time to hypoxia was $5 \mathrm{~h}$, based on our previous studies showing that $5 \mathrm{~h}$ of hypoxia decreased conduction velocity, total $\mathrm{Cx} 43$ protein and the $\mathrm{Cx} 43$ immunofluorescence signal (Zeevi-Levin et al. 2005). At the end of the experiment the cultures were immediately fixed in $4 \%$ paraformaldehyde in PBS for $10 \mathrm{~min}$, and then re-photographed to determine Cx43 locations.

\section{Immunofluorescence measurements in NRVM}

For validation of the colocalization between GFP signal and Cx43, cultures were fixed for 10 min with $4 \%$ paraformaldehyde and permeabilized with Triton X-100 (0.2\% in PBS). Blocking was performed with $10 \%$ goat serum in PBS for $1 \mathrm{~h}$ at $37^{\circ} \mathrm{C}$, and then incubated overnight at $4^{\circ} \mathrm{C}$ with mouseanti-Cx43 (1: 100 dilution), followed by incubation with rhodamine anti-mouse secondary antibody ( $1: 200$ dilution $)$ for $1 \mathrm{~h}$ at room temperature. Cultures were also stained for nuclei (TO-PRO, $1: 500$ dilution). For the hypoxia experiments, confocal microscopy was performed using an upright Nikon Eclipse E600 microscope and Bio-Rad Radiance 2000 scanning system. Cultures were examined by a fluorescence microscopy at a magnification of $40 \times$ using a water immersion lens with numerical aperture of 0.8 , lateral resolution of $0.29 \mu \mathrm{m}$ and depth resolution of $\sim 2 \mu \mathrm{m}$. Areas selected for analysis consisted of well-preserved beating cardiomyocytes expressing Cx43-GFP. Selected cells were marked, and locations were registered in order to re-photograph the same cells after exposure to hypoxia. By so doing, each pair of cells served as its own control. The same optical parameters of magnification, iris dimensions and gain were used in preand post-hypoxia exposures.

\section{Generation of recombinant adenoviruses using the AdEasy System and NRVM infection}

The construct of GFP-tagged Cx43 was kindly provided by Dr. Matthias Falk (Falk 2000) (The Scripps Research Institute, La Jolla, CA, USA). The construct was first cloned into the shuttle vector pAdTrack-CMV, and the resultant plasmid was linearized by digestion with restriction endonuclease Pme I, and subsequently cotransformed into Escherichia coli BJ5183 cells with an adenoviral backbone plasmid, pAdEasy-1. Subsequently, the linearized recombinant plasmid was transfected into the adenovirus packaging cell line HEK 293 cells. Following infection, the generated recombinant adenoviruses were amplified and purified as previously described (He et al. 1998). The am- plification was performed in HEK 293 cells using DMEM $5 \%$ medium. HEK 293 cells were infected by the virus for 24-36 h, until cell lysis was noted. The supernatant was collected and used for infection of larger amount cell plates, until a large amount of viral supernatant was obtained. Viral purification was performed by ultracentrifugation (90 $\mathrm{min}, 35,000 \mathrm{rpm}$, followed by a second round of $20 \mathrm{~h}$ at $35,000 \mathrm{rpm}$ ) in a $\mathrm{CsCl}$ gradient. The viral aspirate underwent desalting of CsCl by using Sephadex G-25 column. The titer was calculated and the viral stock was kept at $-70^{\circ} \mathrm{C}$ until the experiment. The purified recombinant adenoviruses were used for gene transfer into NRVM. Dissociated NRVM were seeded in collagen-coated Petri dishes $\left(\sim 5 \times 10^{4}\right.$ cells $\left./ \mathrm{cm}^{2}\right)$. At this low concentration of cells, the cardiomyocytes are stretched relatively flat on the Petri dishes, form small number of contacts with adjacent cells (2-4 cells), and their borders can be easily examined. The cells were grown in complete basic medium (F10, 5\% FCS, 5\% DHS, P/S, $1 \mathrm{mM} \mathrm{CaCl}_{2}$ and BrdU). After several trials, we determined that the best approach to receive relatively homogenous $\mathrm{Cx} 43-\mathrm{GFP}$ expression is to mix the virus with NRVM before seeding. After $24 \mathrm{~h}$, the viruscontaining medium was replaced with virus-free medium. The MOI (multiplicity of infection) was $\sim 10$. Subsequently, the medium was replaced every other day until the day of the experiment (5-6 days after seeding).

\section{Validation of CX43-GFP expression in NRVM}

Five-day-old cultures expressing Cx43-GFP were fixed in 4\% paraformaldehyde in PBS for 10 min and permeabilized with $0.1 \%$ triton-X in PBS for 10 min. After blocking with goat serum $(1: 10$ in PBS) to suppress nonspecific fluorescence, cultures were incubated with monoclonal mouse anti-rat Cx43 antibodies (Chemicon, Temecula, CA, USA) for $1 \mathrm{~h}$, followed by incubation with goat anti-mouse Cye3 antibodies. Cultures were photographed using a confocal microscope $(\times 40$ oil immersion lens, NA $=1.3)$, and colocalization of the GFP and Cye3 signal (representing the GFP protein and the $\mathrm{Cx} 43$ antibody, respectively) was calculated using LaserSharp software.

\section{Statistical analysis}

The results are presented as mean \pm SEM. Statistical analysis was performed using Sigmastat ${ }^{\mathrm{TM}} 3.0$ software. To analyze the electrophysiological parameters, two-way repeated measure ANOVA was used to compare variables between groups. For comparing the distance of $\mathrm{Cx} 43-\mathrm{GFP}$ spots from the gap junction area (see below), Student paired $t$-test was used. In cases where normality test failed, a Mann-Whitney rank sum test was used. $p<0.05$ was considered statistically significant in all comparisons. 
A

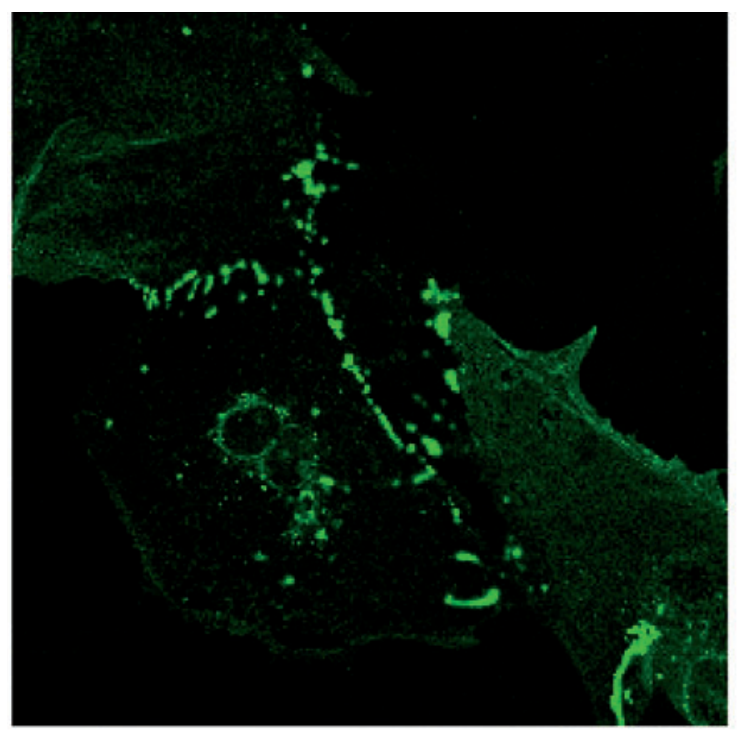

C

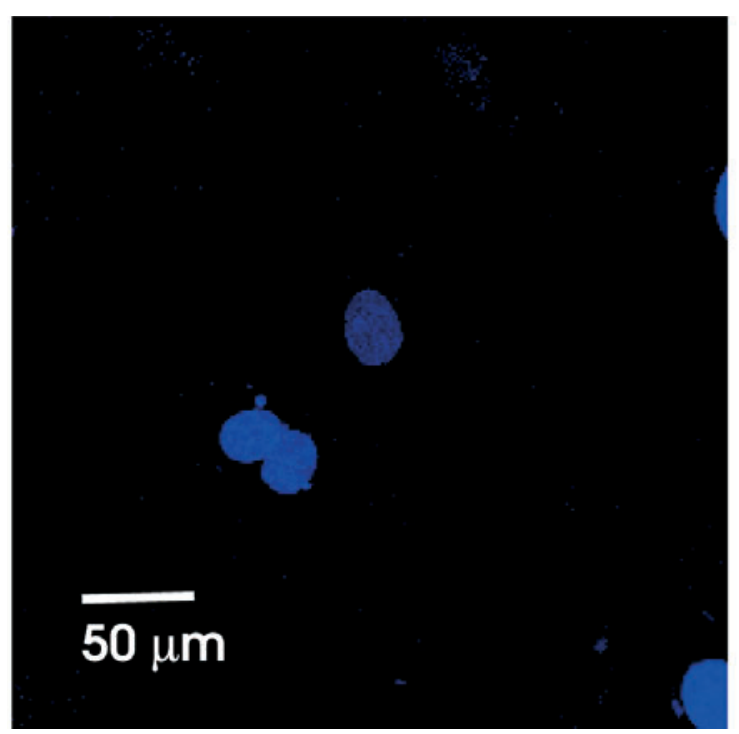

B

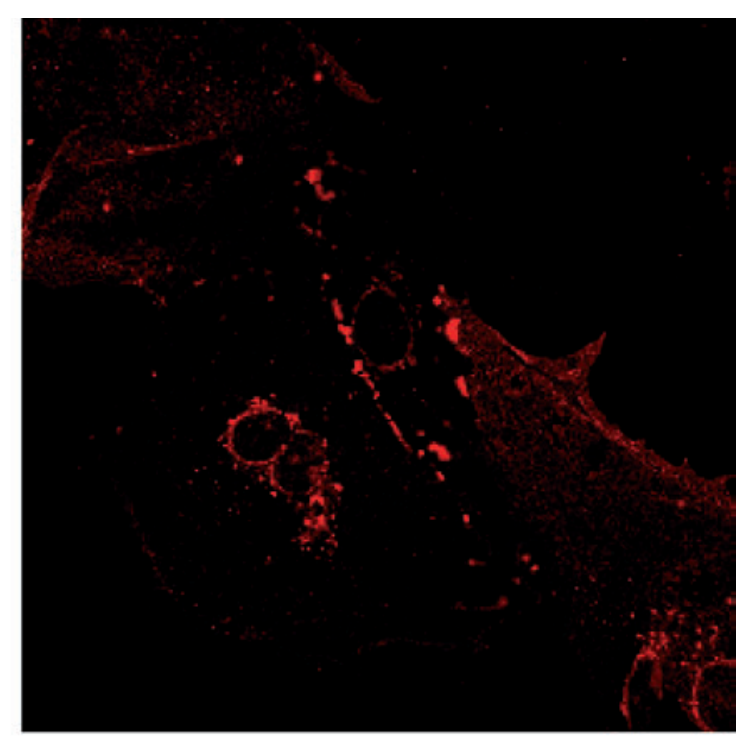

D

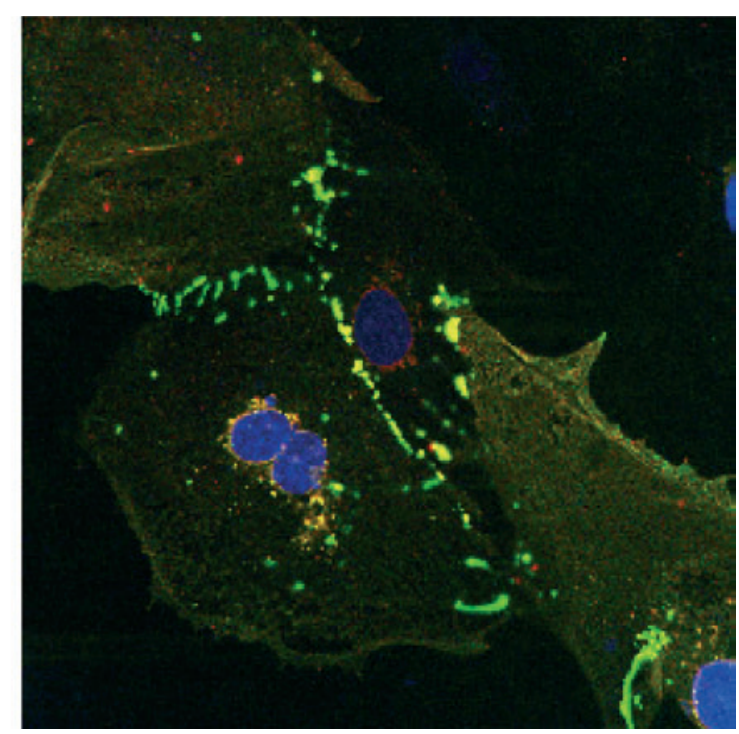

Figure 1. Confocal images of a NRVM culture showing the Cx43-GFP signal and the immunofluorescence staining for the native Cx43. A. The green channel shows the Cx43-GFP signal. The signal represents the GFP protein attached to Cx43. B. The red channel: the signal represents the secondary antibody attached to $\mathrm{Cx} 43$. C. The far-red channel shows the signal from ToPro staining (nuclei). D. A merge of all 3 layers.

\section{Results}

Validation of CX43-GFP expression in NRVM cultures following viral infection

The first requisite of our study was to apply/develop a variety of analytical tools permitting the immunofluorescence tracking of Cx43-GFP in live NRVM. To satisfy this requirement, NRVM cultures underwent viral infection, and Cx43-GFP expression was confirmed using confocal laser microscopy.
Thereafter, the cultures were processed for immunofluorescence analysis, as described in the Methods, and shown in a representative experiment (Fig. 1). To ascertain that the GFP signal (Cx43-GFP) is co-localized with the native Cx43 (determined by immunofluorescence staining), LaserSharp software was utilized, and the following three correlation coefficients were calculated:

1.Pearson correlation coefficient is a common measure of the correlation between two variables (Manders et al. 2003; Comeau et al. 2006; Crawford 2006; Amazit et al. 
2007). It is most accurate when the intensity of the 2 signals is approximately equal, and is calculated according to the following equation:

$$
R_{r}=\frac{\sum_{i}\left(S_{1 i}-S_{1 \text { aver }}\right) *\left(S_{2 i}-S_{2 \text { aver }}\right)}{\sqrt{\sum_{i}\left(S_{1 i}-S_{1 \text { aver }}\right)^{2} * \sum_{i}\left(S_{2 i}-S_{2 a v e r}\right)^{2}}}
$$

where $S_{1}$ is intensity of pixels in the first image and $S_{2}$ is the intensity of pixels in the second image. $S_{1 \text { aver }}$ and $S_{2 \text { aver }}$ are the average intensities of first and second images, respectively.

2. Overlap correlation has a relative insensitivity to differences in signal intensities between various components of an image, and thus is more accurate than Pearson coefficient for unequal signals. The major disadvantage is that the overlap correlation is proportional to the number of co-localized pixels; i.e., two "similar" images with only few enhanced pixels will receive a relatively low score.

$$
R=\frac{\sum_{i} S_{1 i} * S_{2 i}}{\sqrt{\sum_{i}\left(S_{1 i}\right)^{2} * \sum_{i}\left(S_{2 i}\right)^{2}}}
$$

3.The colocalization coefficients M1 and M2. To overcome the dependency of the overlap correlation on the number of the co-localized pixels, the overlap correlation was divided into two coefficients as follows (Eq. 3) (Comeau et al. 2006):

$$
\begin{gathered}
M 1=\frac{\sum_{i} S 1_{i, \text { colc }}}{\sum_{i} S 1} \\
M 2=\frac{\sum_{i} S 2_{i, \text { colc }}}{\sum_{i} S 2}
\end{gathered}
$$

where $S 1_{\mathrm{i}, \text { coloc }}=\mathrm{S} 1_{\mathrm{i}}$ if $\mathrm{S} 2_{\mathrm{i}}>0 ; \mathrm{S} 2_{\mathrm{i}, \text { coloc }}=\mathrm{S} 2_{\mathrm{i}}$ if $\mathrm{S} 1_{\mathrm{i}}>0$.

The values of the 3 co-localization coefficients (Table 1) indicate a high degree of correlation between the GFP and the immuno-labeled (native) $\mathrm{Cx} 43$ signals. In conclusion, the GFP signal reliably represents the presence of the fusion protein consisting of GFP and Cx43, which is preferentially located at the outer cell membrane.

\section{GFP-CX43 viral infection does not affect the electrophysi- ological properties of NRVM cultures}

To determine whether the infection procedure affected the electrophysiological properties of NRVM, electrograms were recorded in the MEA apparatus, and activation maps generated in spontaneously beating control and GFP-Cx43infected cultures (Fig. 2). In these experiments performed on 20 control and 6 transfected cultures, conduction velocity $(25.3 \pm 2.7$ and $23.7 \pm 2.1 \mathrm{~cm} / \mathrm{s})$, the maximal upstroke velocity of the electrogram $\left(\mathrm{dV} / \mathrm{dt}_{\max }=-5.5 \pm 0.7\right.$ and $-5.1 \pm 0.7 \mu \mathrm{V} / \mathrm{s})$, electrogram duration $(6.22 \pm 0.16$ and $6.27 \pm 0.32 \mathrm{~ms})$ and electrogram amplitude (101.41 \pm 5.42 and $104.56 \pm 11.86 \mu \mathrm{V}$ ) were comparable in the two groups. Further, the spontaneous beating rate was similar in the control and Cx43-GFP cultures: control $(n=20): 1.47 \pm 0.23 \mathrm{~Hz}$; GFP-Cx43 $(n=6): 1.46 \pm 0.35 \mathrm{~Hz}(p=0.99)$. Collectively, these results indicate that infecting NRVM with Cx43-GFP did not alter their electrophysiological properties or spontaneous beating rate.

\section{Effect of hypoxia on Cx43 distribution}

After confirming by means of the correlation coefficients that the Cx43-GFP signals co-localizes with native $\mathrm{Cx} 43$, we investigated the effect of hypoxia on $\mathrm{Cx} 43$ distribution. Since the analysis required clear visualization of cell borders, in these experiments NRVM were seeded at a low density, which caused flattening of individual myocytes. To photograph the same cells before and after exposure to hypoxia, a grid was drawn on the bottom of the Petri dish, enabling accurate marking of the cell location.

The first step in these experiments was to infect NRVM with Cx43-GFP. Subsequently, the expression of Cx43-GFP was confirmed, and beating myocytes with well-defined boundaries were photographed and their exact location marked. Next, the cultures were transferred to the hypoxia incubator for $5 \mathrm{~h}$, and then the same myocytes were rephotographed using the same optical parameters (Fig. 3). As seen in these two pairs of myocytes, a strong GFP signal is confined to the border area between adjacent cells. To determine whether hypoxia causes migration of $\mathrm{Cx} 43$ away from the border area into the cell interior, a MATLAB semi-automatic tool was developed, as follows: Cx43-GFP locations are represented by the highlighted pixels, but maximally saturated pixels may represent different amounts of Cx43. Therefore, the intensity of the pixels was not taken into consideration. The analytical process was as follows:

Table 1. Statistical analysis of the correlation coefficients of the colocalization between the GFP-Cx43 signal and the immunolabeled $\mathrm{Cx} 43$

\begin{tabular}{lcc}
\hline & Mean value & S.E.M. \\
\hline Pearson coefficient & 0.79 & 0.047 \\
Overlap R coefficient & 0.855 & 0.034 \\
M1 colocalization coefficient & 0.969 & 0.035 \\
M2 colocalization coefficient & 1 & 0 \\
\hline
\end{tabular}

A high degree of matching was observed for all methods of comparison ( $n=6$ cultures), between the GFP signal and the immunolabeled $\mathrm{Cx} 43$. 
A

B

\section{Control}

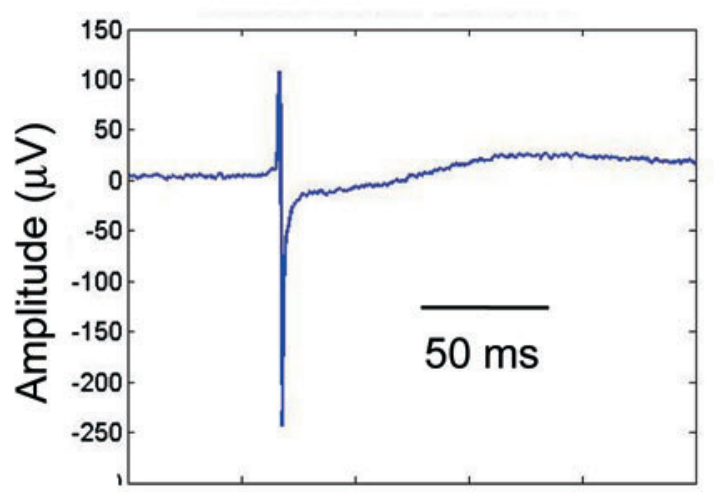

C

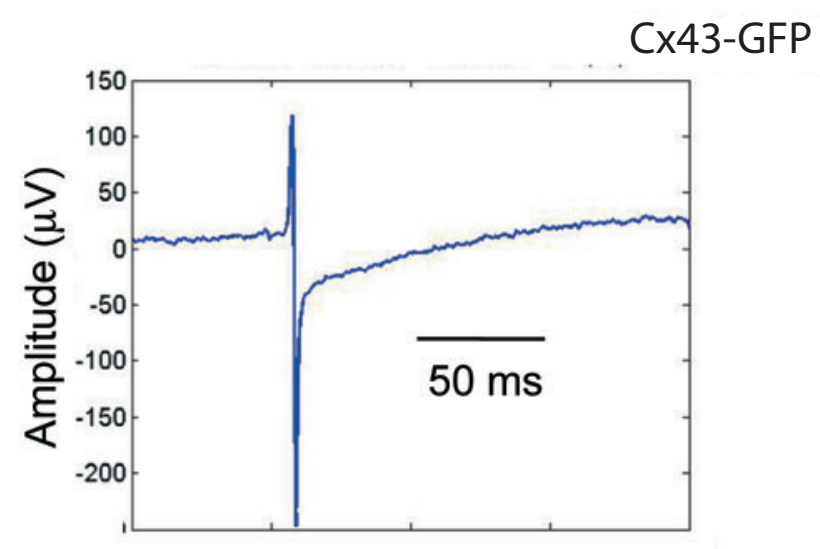

D

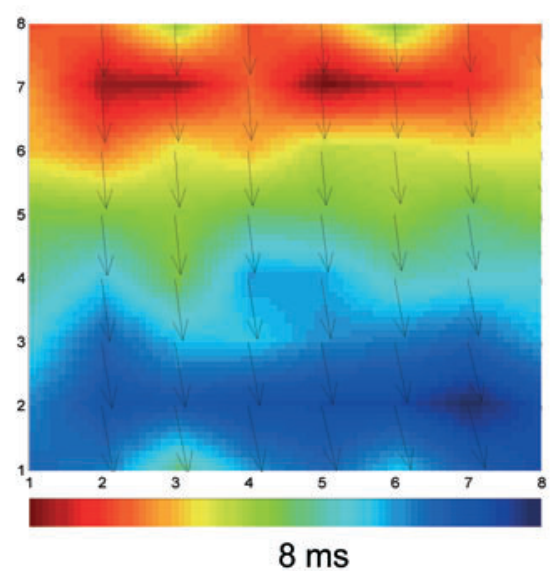

$8 \mathrm{~ms}$

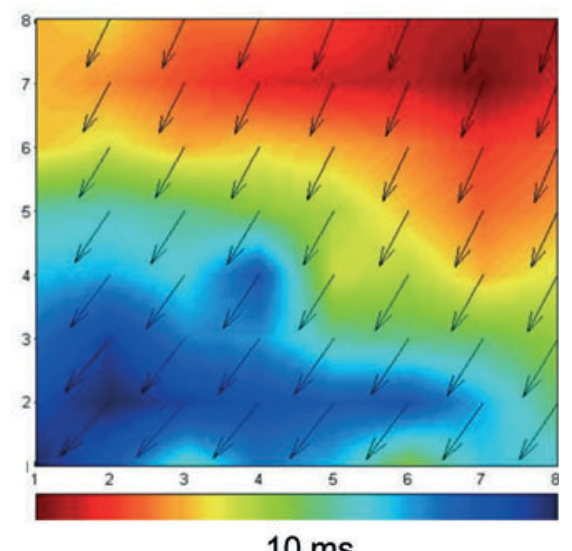

Figure 2. Representative electrograms and activation maps from control and Cx43-GFP transfected cultures. A and C. Electrograms recorded from a control and a Cx43-GFP infected culture. B and D. Activation maps generated in a control culture and in a Cx43-GFP infected culture (see Methods for details). The red represents early activation and blue late activation (see bar scale time below the map). In $\mathrm{B}$ and $\mathrm{D}$, conduction velocity was $18.7 \mathrm{~cm} / \mathrm{s}$ and $22.2 \mathrm{~cm} / \mathrm{s}$, respectively.

initially, the border between two myocytes was marked by the operator in the pre-hypoxia image, using three points that determine the equation of a circle (Fig. 4A and C). Subsequently, the distance of the Cx43-GFP signal from the border was automatically calculated using Eq. (4):

$$
\text { Distance } \left.=\operatorname{abs}\left(\left((X i-C x)^{2}+(Y i-C y)^{2}\right)\right)^{1 / 2}-R\right)
$$

where $C x$ and $C y$ are the coordinates of the circle center, and $R$ is its radius. $X i$ and $Y i$ are the $\mathrm{Cx} 43$ coordinates. An absolute value is used in the Eq. (4) since the inner expression can be positive or negative, depending whether the $\mathrm{Cx} 43$ GFP spot is inside or outside the circle, while the distance from the circle is a positive number by definition. We defined a maximal distance $L ; C x 43-G F P$ spots which are closer than
$L$ to the circle are considered close to the border, while spots which are further away than $L$ are considered outside the border area. $L$ is calculated by Eq. (5):

$$
L=\text { Mean distance }+2 \cdot \text { Standard deviations }
$$

The border area was defined to include all the areas within the distance $L$ from the borderline. This definition includes approximately $95 \%$ of the Cx43-GFP signals within the border zone. After defining the border line and the border zone in the pre-hypoxia image (Fig. $4 \mathrm{~A}$ and C), the posthypoxia image was analyzed using the same definitions (Fig. 4B and D). By so doing, we were able to compare the pre-hypoxia to the post-hypoxia number of non-border Cx43-GFP spots (spots at a distance from the border that is 
A

\section{Pre-hypoxia}

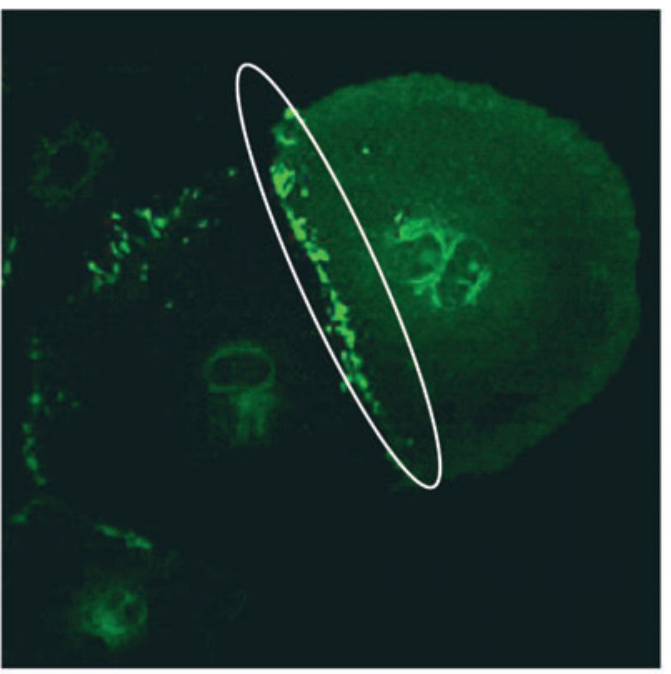

C

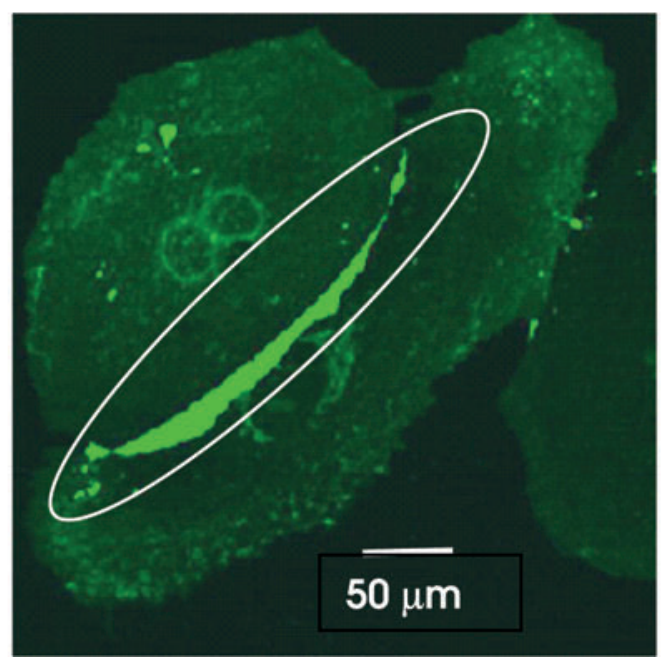

B

Post-hypoxia

D
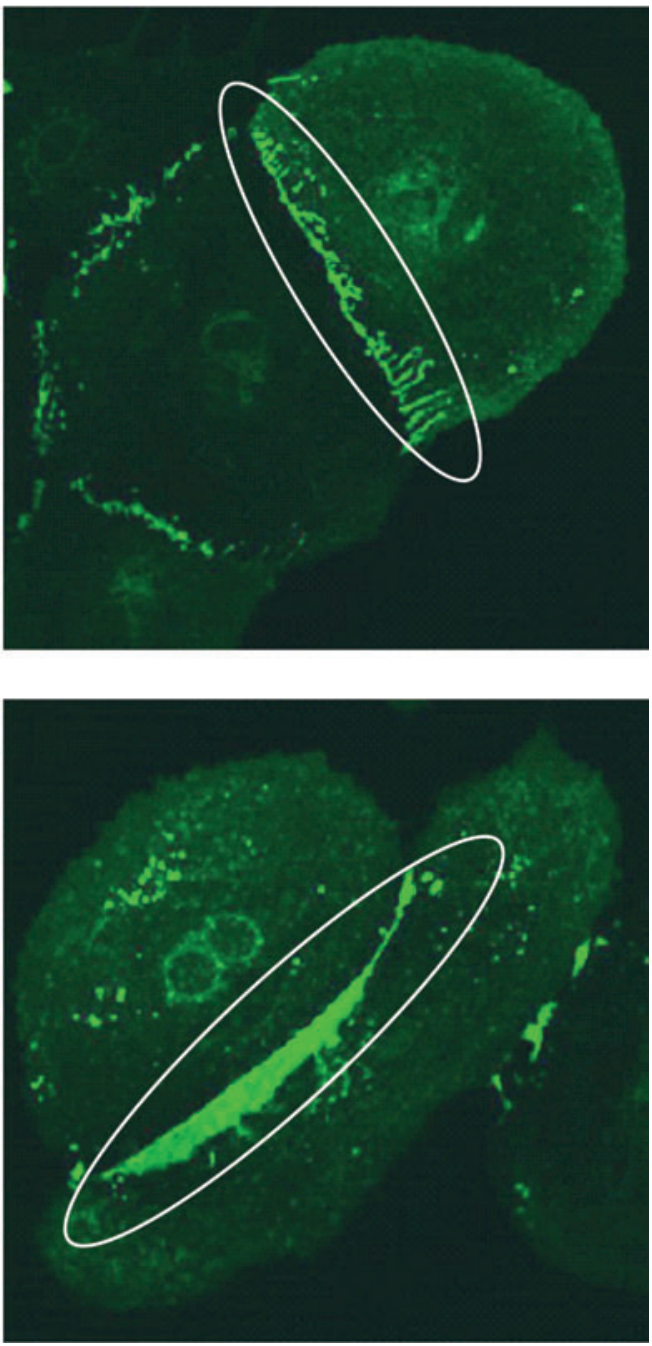

Figure 3. The effect of hypoxia on GFP-Cx43 distribution in two pairs of cardiomyocytes. A and C. Pre-hypoxia images of two different pairs of cardiomyocytes expressing GFP-Cx43, from experiments No. 1 and 2 (see Table 2), respectively. B and D. Post-hypoxia images of the same cells obtained after $5 \mathrm{~h}$ of hypoxia. It can be seen that in both cell pairs, the strongly-stained boundary areas (encircled by the white ellipsoid) are broader after hypoxia.

larger than $L$; see Eq. (5)). Indeed, as seen by the histogram analysis in a representative experiment, the shape of the histogram in the post-hypoxia state declines less sharply than the pre-hypoxia histogram (Fig. 5). This phenomenon indicates that in the latter more spots are located closer to the border, as well as that $\mathrm{Cx} 43$ distribution is gradual process. To evaluate this phenomenon mathematically, we defined $x$ as the distance from the borders that includes $66 \%$ of the non-border Cx43-GFP spots. In the pre- and post-hypoxia states, $x$ (mean \pm SEM) was $20.3 \pm 9.7$ and $25.5 \pm 7.6$ pixels ( $n=9$; $t$-test, $p=0.014)$, respectively. This calculation has the advantage that it eliminates any effect of a significant number of perinuclear Cx43-GFP spots that might be present due to an over-expression of the virus.

Finally, to provide a quantitative measure of the Cx43GFP migration from the gap junctional areas into the cell interior, we compared in 9 pairs of myocytes, the number of border vs. non-border Cx43-GFP spots, and the distance distribution of Cx43-GFP spots from the border before and after the induction of hypoxia. To determine the changes in Cx43-GFP distribution, the analysis included only spots outside the border zone. Hence, an analysis that considers only non-border spots may achieve more accurate results since many of the in-border spots are represented by fully 
A

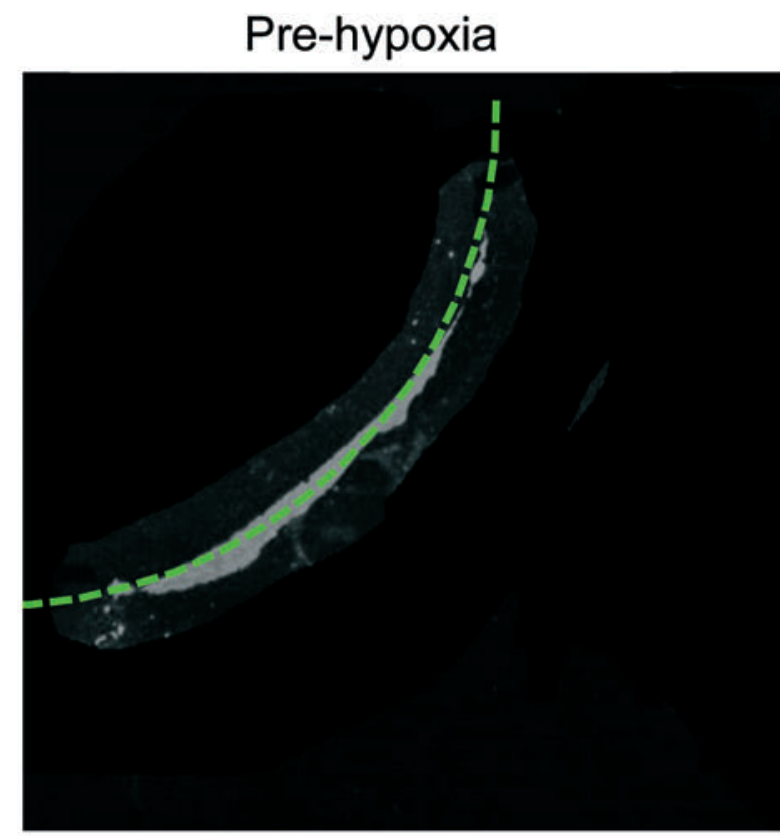

C

\section{Pre-hypoxia image with border and non-border Cx43-GFP}

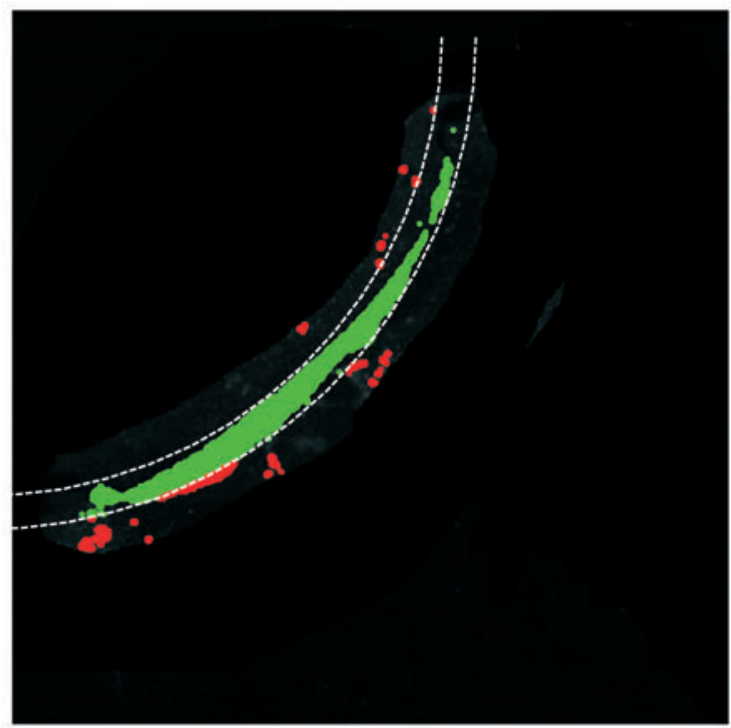

\section{- 4172 Border Cx43-GFP \\ - 469 Non-border Cx43-GFP}

B Post-hypoxia

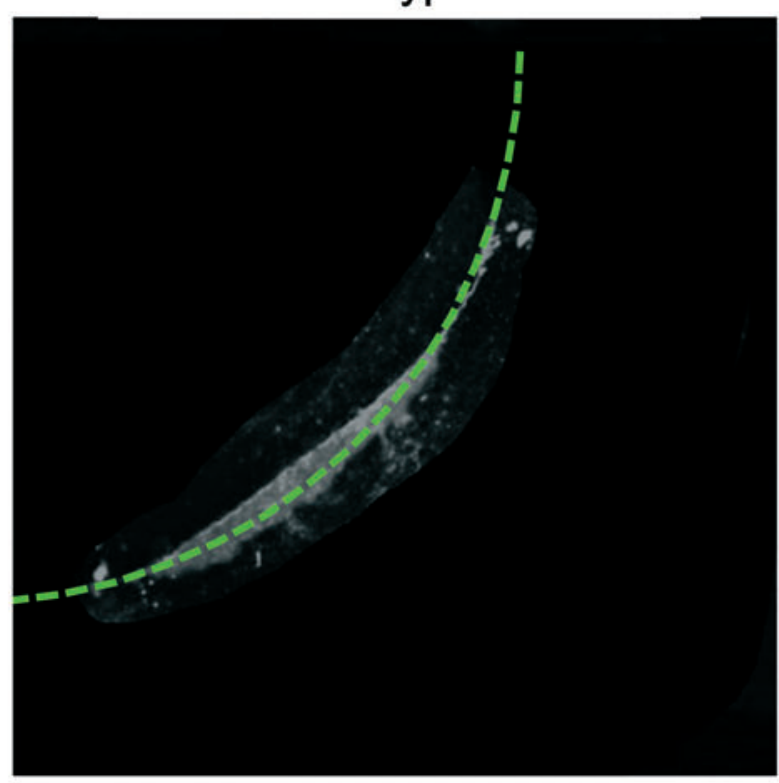

D

Post-hypoxia image with border and non-border Cx43-GFP

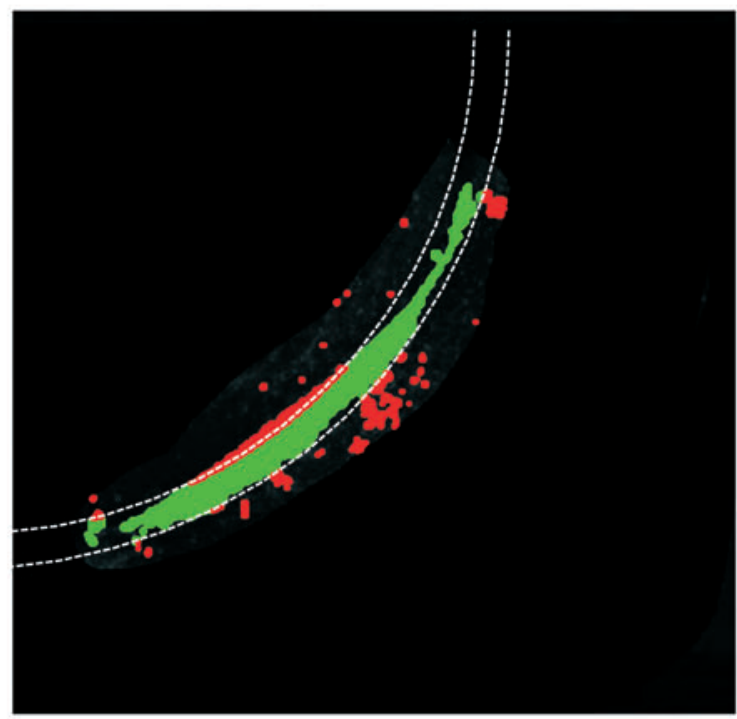

- 5249 Border Cx43-GFP

- 1233 Non-border Cx43-GFP

Figure 4. A representative example of the analysis of border and non-border Cx43-GFP distribution before and after hypoxia in experiment No. 2. A. The border between the 2 cells was marked as a circle line (green color) in the pre-hypoxia state. B. The same circled border line was employed in the post-hypoxia state. Subsequently, calculation of the border and non-border Cx43-GFP spots was performed, as described in the text. C and D. Pre- and post-hypoxia images, respectively, illustrating the border area. The green dots represent the border Cx43-GFP, and the red dots the non-border Cx43-GFP. The border area is marked by the 2 dashed white lines. 


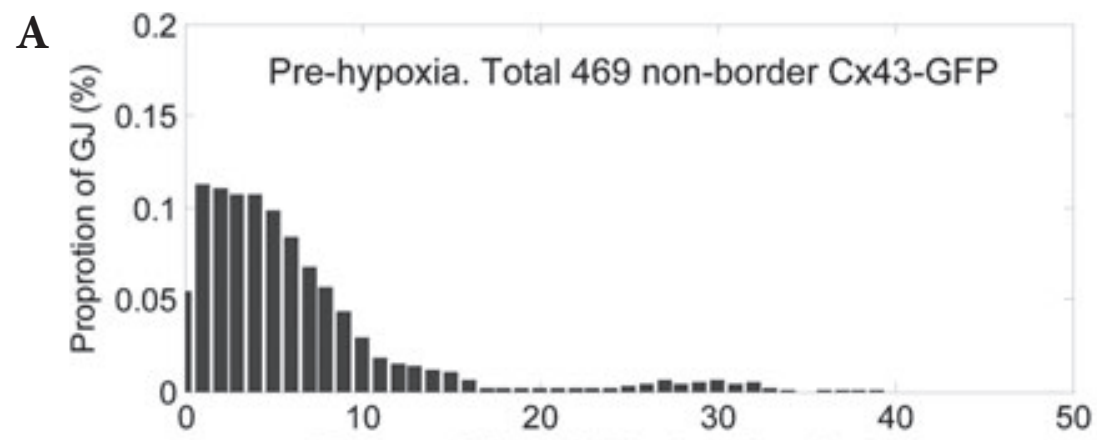

Distance of Cx43-GFP in pixels from the border

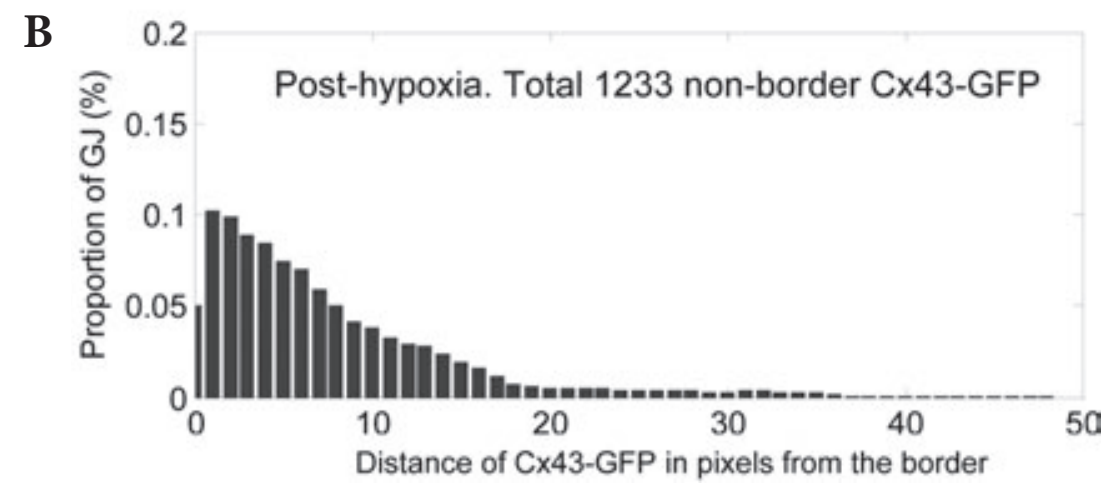

Figure 5. Histograms of Cx43-GFP spots distance distribution derived from experiment No. 2. A and B. illustrate the Cx43-GFP distance distribution before and after exposure to hypoxia, respectively. In this experiment, 469 non-border Cx43-GFP spots were present in the pre-hypoxia state, compared to 1233 in the post-hypoxia state.

saturated pixels, which can contain large and unknown numbers of Cx43-GFP molecules. In this regards, Table 2 shows the mean distance of non-border Cx43-GFP spots from the border in the 9 experiments performed. In 6 experiments the mean distance of non-border gap junctions was higher post-hypoxia, while in the other 3 experiments, there was no statistically significant difference. A binomial test was used to determine whether these results are statistically significant;

Table 2. Comparison of the mean distance of non-border Cx43-GFP spots from the border, between pre- and post-hypoxia NRVM cultures

\begin{tabular}{crcrcr}
\hline \multirow{2}{*}{ Experiment No. } & \multicolumn{2}{c}{ Pre-hypoxia } & \multicolumn{2}{c}{ Post-hypoxia } & \multirow{2}{*}{$p$} \\
\cline { 2 - 5 } & $n$ & Mean distance & $n$ & Mean distance & 0.031 \\
2 & 9 & 14.75 & 333 & 24.02 & 0.018 \\
3 & 4 & 22.29 & 109 & 28.98 & 0.251 \\
4 & 248 & 16.41 & 594 & 15.48 & $<0.001$ \\
5 & 0 & 0 & 53 & 12.76 & 0.002 \\
6 & 64 & 18.64 & 257 & 20.10 & 0.296 \\
7 & 19 & 34.13 & 17 & 34.71 & $<0.001$ \\
8 & 212 & 30.13 & 86 & 27.64 & 0.997 \\
9 & 19 & 28.22 & 783 & 23.67 & $<0.001$ \\
\hline
\end{tabular}

In 6 experiments (where Mann-Whitney test with $p<0.05$ was considered significant) the mean distance of non-border Cx43-GFP from the border was larger after exposure to hypoxia. In the remaining 3 experiments (No. $3,6,8$ ), no significant difference was found. $n$, the number of non-border Cx43-GFP spots. 
this test is more appropriate than the Student $t$-test since few "very positive" experiments can cause a bias. In addition, it is thought that the Cx43-GFP internalization has the same effect on conduction velocity regardless of the mean distance of the internalization. In summary, the number of non-border Cx43-GFP spots was significantly higher posthypoxia ( $p=0.038$ for comparison of non-border $\mathrm{Cx} 43$-GFP spots number; $p=0.066$ for Student $t$-test (2-tailed $t$-test; $p=0.04)$ ). Since the analysis of the mean distances from the border can underestimate the number of Cx43-GFP spots, a comparison of the value of $n \times$ mean distance, which takes into account only the non-border Cx43-GFP spots, was performed. This value may better represent the Cx43-GFP internalization in response to hypoxia. As shown in Fig. 6 this value was significantly $(p<0.05)$ higher after exposure to hypoxia. Based on these experiments, we concluded that in this model hypoxia caused migration of $\mathrm{Cx} 43$ from the gap junction area into the cell interior, an effect which will contribute to the reduction in conduction velocity under hypoxic conditions.

\section{Discussion}

The present study provides evidence supporting the hypothesis that hypoxia causes $\mathrm{Cx} 43$ internalization, thus contributing to the reduction in conduction velocity by hypoxic/ischemic conditions. A critical difference between this and earlier studies which analyzed control and hypoxic cultures separately is that we determined the effect of hypoxia on Cx43 by monitoring the Cx43-GFP signal in the same culture before and after hypoxia.

\section{Experimental model}

Monitoring individual proteins in living cells has become feasible due to the development of gene transfer techniques, and by coupling the protein of interest to a fluorescence label (e.g., GFP or YFP) (Jordan et al. 1999; Gaietta et al. 2002). Importantly, in the present study we used a cytomegalovirus promoter, which with the use of adenovirus resulted in over-expression of $\mathrm{Cx} 43$, which in turn might affect the electrophysiological properties of the cardiomyocytes. Nonetheless, as shown in Fig. 2, activation and conduction properties were unaffected. These findings agree with previous reports in NRVM demonstrating that only a relatively large decrease $(\sim 50 \%)$ in the Cx43 amount affected conduction velocity (Rohr et al. 1997, 2004). In contrast, in NRVM, 6 h of pulsatile stretch caused dramatic upregulation of Cx43 (the Cx43 signal increased from 0.73 to $2.02 \%$ of the cell area) paralleled by an increase in conduction velocity from $27 \mathrm{~cm} / \mathrm{s}$ to $37 \mathrm{~cm} / \mathrm{s}$ (Zhuang et al. 2000). Based on these and on our own findings, it is reasonable to conclude that this in

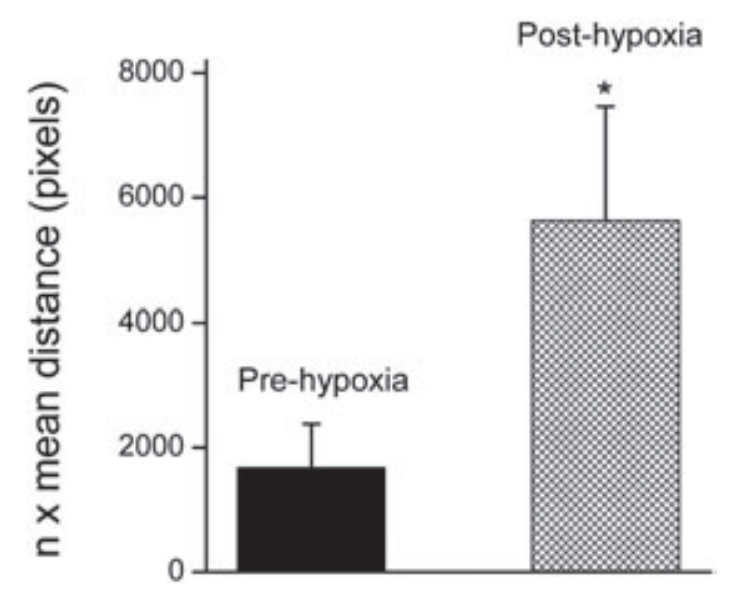

Figure 6. Comparison of the product of $n \times$ mean distance of nonborder Cx43-GFP from the border between cells, before and after hypoxia. After exposure to $5 \mathrm{~h}$ of hypoxia, the $n \times$ mean distance of non-border (from the border) Cx43-GFP was significantly higher than in pre-hypoxia $\left(n=9,{ }^{*} p<0.05\right)$.

vitro experimental setting is suitable for testing the hypothesis that hypoxia causes $\mathrm{Cx} 43$ internalization.

\section{Hypoxia causes Cx43 internalization}

To provide a more rigorous support to the hypothesis that hypoxia causes migration of $\mathrm{Cx} 43$ from the gap junctional area into the cell interior, we developed the means to monitor in the same pair of cardiomyocytes, the changes in Cx43-GFP distribution within the border and the non-border areas. The first step was to ascertain that the Cx43-GFP signal indeed represents the native $\mathrm{Cx} 43$. As indicated by the 3 calculated coefficient correlations, the native $\mathrm{Cx} 43$ (represented by the immunolabeled $\mathrm{Cx} 43$ protein) and the GFP signals were co-localized. Next, to determine quantitatively whether Cx43-GFP molecules migrated away from the gap junctional areas, we defined for every cell pair a "border area" and a "non-border" area; this procedure enabled us to count (using a MATLAB routine) the number of GFP-Cx43 hot spots before and after hypoxia, thus providing an objective means to assess $\mathrm{Cx} 43$ internalization. Using the border and non-border definitions, as shown in Table 2, the mean distance of non-border Cx43-GFP spots was higher post-hypoxia. To further estimate $\mathrm{Cx} 43$ GFP internalization, we calculated the value of $n x$ mean distance ( $n=$ number of non-border hot spots), which was much higher after exposure to hypoxia. Collectively, these findings support the hypothesis that in this experimental model hypoxia caused $\mathrm{Cx} 43$ internalization, an effect which can contribute to the decreased conduction velocity by hypoxic/ischemic conditions. 


\section{Effects of hypoxia on Cx43}

Several studies reported that hypoxia decreases conduction velocity, and proposed that this can result from decreased gap junctional conductance, a decrease in the content of the Cx43 protein, a shift from the phosphorylated to the nonphosphorylated state and/or Cx43 translocation (Beardslee et al. 2000; Jain et al. 2003; Zeevi-Levin et al. 2005; Zhang et al. 2006). For example, ischemia caused $\mathrm{Cx} 43$ dephosphorylation and accumulation of non-phosphorylated $\mathrm{Cx} 43$ within the gap junctions (Beardslee et al. 2000). Importantly, these authors also demonstrated that hypoxia caused translocat of Cx43 into cellular pods, an observation based on Western blot analysis (which represents the total amount of $\mathrm{Cx} 43$ in the cell) showing similar amounts of $\mathrm{Cx} 43$ in the pre-hypoxic and post-hypoxic cultures, while the immunoflourescence signal at the cell membrane was decreased. Hence, in contrast to our study, Beardslee and co-workers provided an indirect support for the hypothesis that $\mathrm{Cx} 43$ undergoes internalization during hypoxia. In this regards and in partial disagreement with Beardslee et al., we found that after $5 \mathrm{~h}$ of hypoxia in NRVM, total Cx43 protein was decreased by $50 \%$, while the non-phosphorylated Cx43 isoform was unchanged. Confocal analysis showed a $55 \%$ decrease in the gap junctional Cx43 fluorescence signal, a 55\% decrease in gap junction number and a $26 \%$ decrease in size. The reduction in $\mathrm{Cx} 43$ protein levels was associated with an approximately $20 \%$ decrease in conduction velocity compared to normoxic cultures (Zeevi-Levin et al. 2005).

\section{Study limitations}

Although we showed that hypoxia causes Cx43-GFP internalization, there are several limitations to this technique of monitoring the infected Cx43-GFP signal: 1) the new constructed gene has a powerful promoter thus increasing the amount of $\mathrm{Cx} 43$ produced, which may change the electrical properties of NRVM. However, as shown in Fig. 2, at the network level, propagation and activation did not differ between control and infected cultures. 2) The biophysical characteristics of $\mathrm{Cx} 43-\mathrm{GFP}$ may differ from the native $\mathrm{Cx} 43$. Specifically, while the Cx43-GFP channel seems to lack the low-conductance state seen in the native channel (Bukauskas et al. 2001), the assembly and transport of Cx43-GFP molecule are similar to those of the native channel (Jordan et al. 1991; Falk 2000; Lauf et al. 2002).

In summary, in the present study we tested the hypothesis that hypoxia causes Cx43 internalization, using in situ confocal microscopy to monitor NRVM transfected with Cx43-GFP, with each culture serving as its own control. In support of this hypothesis, we found that compared to normoxia, $5 \mathrm{~h}$ of hypoxia caused a reduction of the Cx43GFP signal at the gap junctional areas (border area) and an increase in the Cx43-GFP signal in the non-border areas, thus providing an additional explanation for the intercellular uncoupling during ischemic conditions.

Acknowledgement. This work was supported by the US Israel Binational Science Foundation (BSF), the German Israeli Foundation (GIF) and the Rappaport Family Institute for Research in the Medical Sciences.

\section{References}

Amazit L., Pasini L., Szafran A. T., Berno V., Wu R. C., Mielke M., Jones E. D., Mancini M. G., Hinojos C. A., O’Malley B. W., Mancini M. A. (2007): Regulation of SRC-3 intracompramental dynamics by estrogen receptor and phosphorylation. Mol. Cell. Biol. 27, 6913-6932; doi:10.1128/MCB.01695-06

Ausma J., van der Velden H. M., Lenders M. H., van Ankeren E. P., Jongsma H. J., Ramaekers F. C., Borgers M., Allessie M. A. (2003): Reverse structural and gap-junctional remodeling after prolong atrial fibrillation in the goat. Circulation 107, 2051-2058; doi:10.1161/01. CIR.0000062689.04037.3F

Beardslee M. A., Lerner D. L., Tadros P. N., Laing J. G., Beyer E. C., Yamada K. A., Kléber A. G., Schuessler R. B., Saffitz J. E. (2000): Dephosphorylation and intracellular redistribution of ventricular connexin 43 during electrical uncoupling induced by ischemia. Circ. Res. 87, 656-662

Bukauskas F. F., Bukauskiene A., Bennett M. V., Verselis V. K. (2001): Gating properties of gap junction channels assembled from connexin 43 and connexin 43 with green fluorescent protein. Biophys. J. 81, 137-152; doi:10.1016/S00063495(01)75687-1

Chaldoupi S. M., Loh P., Hauer R. N., de Bakker J. M., van Rijen H. V. (2009): The role of connexin 40 in atrial fibrillation. Cardiovasc. Res. 84, 15-23; doi:10.1093/cvr/cvp203

Crawford S. L. (2006): Statistical primer for cardiovascular disease: correlation and regression. Circulation 114, 2083-2088; doi:10.1161/CIRCULATIONAHA.105.586495

Comeau J. W. D., Constatino S., Wiseman P. W. (2006): A guide to accurate fluorescence microscopy colocalization measurements. Biophys. J. 91, 4611-4622; doi:10.1529/ biophysj.106.089441

Falk M. M. (2000): Connexin-specific distribution within gap junctions revealed in living cells. J. Cell. Sci. 113, 4109-4120

Gaietta G., Deerinck T. J., Adams S. R., Bouwer J., Tour O., Laird D. W., Sosinsky G. E., Tsien R. Y., Ellisman M. H. (2002): Multicolor and electron microscopic imaging of connexin trafficking. Science 296, 503-507; doi:10.1126/science. 1068793

He T. C., Zhou S., da Costa L. T., Yu J., Kinzler K. W., Vogelstein B. (1998): A simplified system for generating recombinant adenoviruses. Proc. Natl. Acad. Sci. U.S.A. 95, 2509-2514; doi:10.1073/pnas.95.5.2509

Jain S. K., Schuessler R. B., Saffitz J. E. (2003): Mechanism of delayed electrical uncoupling induced by ischemic preconditioning. Circ. Res. 92, 1138-1144; doi:10.1161/01. RES.0000074883.66422.C5 
Jordan K., Solan J. L., Dominguez M., Sia M., Hand A., Lampe P. D., Laird D. W. (1991): Trafficking, assembly, and function of a connexin43-green fluorescent protein chimera in live mammalian cells. Mol. Cell. Biol. 10, 2033-2050

Lauf U., Giepmans B. N., Lopez P., Braconnot S., Chen S. C., Falk M. M. (2002): Dynamic trafficking and delivery of connexons to the plasma membrane and accretion to gap junction in living cells. Proc. Natl. Acad. Sci. U.S.A. 99, 10446-10451; doi:10.1073/pnas.162055899

Manders E. M., Stap J., Brakenhoff G. J., van Driel R., Aten J. A. (2003): Dynamics of the three-dimensional replication patterns during the S-phase, analyzed by double labeling of DNA and confocal microscopy. J. Cell. Sci. 103, 857-862

Meiry G., Reisner Y., Feld Y., Goldberg S., Rosen M., Ziv N., Binah O. (2001): Evolution of action potential propagation and repolarization in cultured neonatal rat ventricular myocytes. J. Cardiovasc. Electrophysiol. 12, 1269-1277; doi:10.1046/j.1540-8167.2001.01269.x

Polontchouk L., Haefliger J. A., Ebelt B., Schaefer T., Stuhlmann D., Mehlhorn U., Kuhn-Regnier F., De Vivie E. R., Dhein S. (2001): Effects of chronic atrial fibrillation on gap junction distribution in human and rat Atria. J. Am. Coll. Cardiol. 38, 883-891; doi:10.1016/S07351097(01)01443-7

Reisner Y., Meiry G., Zeevi-Levin N., Barac D. Y., Reiter I., Abassi Z., Ziv N., Kostin S., Schaper J., Rosen M. R., Binah O. (2009): Impulse conduction and gap junctional remodelling by endothelin- 1 in cultured neonatal rat ventricular myocytes. J. Cell. Mol. Med. 13, 562-573; doi:10.1111/ j.1582-4934.2008.00361.x

Rohr S., Kucera J. P., Fast V. G., Kléber A. G. (1997): Paradoxical improvement of impulse conduction in cardiac tissue by partial cellular uncoupling. Science $\mathbf{2 7 5}, 841-844$; doi:10.1126/science.275.5301.841

Rohr S., Kucera J. P., Kleber A. G. (1998): Slow conduction in cardiac tissue, I. effects of a reduction of excitability versus a reduction of electrical coupling on microconduction. Circ. Res. 83, 781-794
Rohr S. (2004): Role of gap junction in the propagation of cardiac action potential. Cardiovasc. Res. 62, 309-322; doi:10.1016/j.cardiores.2003.11.035

Rubin Y., Kessler-Icekson G., Navon G. (1995): The effect of furosemide on calcium ion concentration in myocardial cells. Cell Calcium 18, 135-139; doi:10.1016/01434160(95)90004-7

Saffitz J. E., Laing J. G., Yamada K. A. (2000): Connexin expression and turnover: implication for cardiac excitability. Circ Res. 86, 723-728

Sosunov E. A., Anyukhovsky E. P., Hefer D., Rosen T. S., Danilo P. Jr., Janse M. J., Rosen M. R. (2005): Region-specific, pacing-induced changes in repolarization in rabbit atrium: an example of sensitivity to the rare. Cardiovasc. Res. 67, 274-282; doi:10.1016/j.cardiores.2005.04.009

Spach M. S., Heidlage J. F., Dolber P. C., Barr R. C. (2000): Electrophysiological effects of remodeling cardiac gap junctions and cell size: experimental and model studies of normal cardiac growth. Circ. Res. 86, 302-311

Turner M. S., Haywood G. A., Andreka P., You L., Martin P. E., Evans W. H., Webster K. A., Bishopric N. H. (2004): Reversible connexin 43 dephosphorylation during hypoxia and reoxygenation is linked to cellular ATP levels. Circ. Res. 95, 726-733; doi:10.1161/01.RES.0000144805.11519.1e

Zeevi-Levin N., Barac Y. D., Reisner Y., Reiter I., Yaniv G., Meiry G., Abassi Z., Kostin S., Schaper J., Rosen M. R., Resnick N., Binah O. (2005): Gap junctional remodeling by hypoxia in cultured neonatal rat ventricular myocytes. Cardiovasc. Res. 66, 64-73; doi:10.1016/j.cardiores.2005.01.014

Zhang Y., Kakinuma Y., Ando M., Katare R. G., Yamasaki F., Sugiura T., Sato T. (2006): Acetylcholine inhibits the hypoxia-induced reduction of connexin 43 protein in rat cardiomyocytes. J. Pharmacol. Sci. 101, 214-222; doi:10.1254/jphs.FP0051023

Zhuang J., Yamada K. A., Saffitz J. E., Kléber A. G. (2000): Pulsatile stretch remodels cell-to-cell communication in cultured myocytes. Circ. Res. 87, 316-32

Received: October 8, 2009

Final version accepted: March 9, 2010 